\title{
Quantum dynamics of chiral fermions in a model with anomalous breaking of gauge invariance
}

\author{
H. O. Girotti, ${ }^{*}$ H. J. Rothe, and K. D. Rothe \\ Institut für Theoretische Physik der Universität Heidelberg Philosophenweg 16, D-6900 Heidelberg, West Germany
}

(Received 21 February 1986)

\begin{abstract}
We study the quantum dynamics of chiral fermion fields minimally coupled to a gauge field. The model, originally proposed by Jackiw and Rajaraman, is known to exhibit the anomalous breaking of gauge invariance, which leads to the appearance of an arbitrary parameter $a$. Both functional and operator techniques are used to obtain the two-point fermion Green's functions for $a>1$ and $a=1$. In both cases clustering holds, and the theory contains asymptotically free fermions. The quantum equation of motion for the field tensor resembles formally that of the Proca theory, but with a dynamically generated mass and a nonconserved source. It is found that for $a=1$ the generating functional cannot be written in terms of a manifestly Lorentz-invariant Lagrangian.
\end{abstract}

\section{INTRODUCTION}

In a recent paper $^{1}$ we studied the canonical quantization of a two-dimensional chiral model exhibiting anomalous breaking of gauge invariance. This model was originally proposed by Jackiw and Rajaraman. ${ }^{2}$ At classical level the model is described by the Lagrangian density ${ }^{3}$

$$
\mathscr{L}=-\frac{1}{4} F_{\mu \nu} F^{\mu \nu}+\bar{\psi}\left[i \partial+e \sqrt{\pi} A\left(1+\gamma_{5}\right)\right] \psi .
$$

Much as in the case of the Schwinger model, the fermionic determinant can be computed exactly and the resulting nonlocal theory can again be recast into a local form with the help of a scalar field $\phi(x)$, as shown in Ref. 2. The corresponding effective Lagrangian reads

$$
\begin{aligned}
\mathscr{L}_{\mathrm{eff}}= & -\frac{1}{4} F_{\mu \nu} F^{\mu \nu}+\frac{1}{2}\left(\partial_{\mu} \phi\right)\left(\partial^{\mu} \phi\right) \\
& +e\left(g^{\mu \nu}-\epsilon^{\mu \nu}\right) \partial_{\mu} \phi A_{\nu}+\frac{1}{2} a e^{2} A_{\mu} A^{\mu},
\end{aligned}
$$

where $a$ is an arbitrary real parameter not uniquely determined by the different regularization procedures used for calculating the fermionic determinant. As was pointed out in Ref. 2, no choice of $a$ can restore the original gauge symmetry. Nevertheless the theory remains consistent. ${ }^{2,1}$

As we have shown, ${ }^{1}$ the quantum field theories (1.2) parametrized by $a$ fall into two classes characterized by different constraint structures. Indeed, while for $a \neq 1$ the theory contains two second-class constraints, the number of constraints doubles for $a=1$. Of course, the constraints are always second class, which reflects the fact that the local gauge symmetry is broken irrespective of the value of $a$. The operator solutions $\left(A^{\mu}, \phi\right)$ to the Hamiltonian equations of motion, corresponding to (1.2), were discussed in detail for all $a$ in Ref. 1 .

The main purpose of this paper is to study the quantum dynamics of the fermion field operators implied by the effective Lagrangian (1.2), a problem that was left out of consideration in all previous analyses ${ }^{1,2}$ of the model. To this end we shall use both functional and operator techniques.

In Sec. II we construct the configuration space path- integral representation for the generating functional $Z_{a}$ of all $\left(\psi\right.$ and $A^{\mu}$ ) Green's functions of the theory. The starting point is provided by the corresponding phase-space path integral for systems with second-class constraints, ${ }^{4,5}$ which for this model turns out to be essential. While for $a>1$ one is led for $Z_{a}$ to the standard configuration space path integral in terms of $L_{\text {eff }}$, the same is not true for $a=1$. In fact, although the configuration space representation for $Z_{a=1}$ exists, it cannot be written in terms of a Lorentz-invariant Lagrangian density. This reflects the fact that, for this value of $a$, the matrix $\delta^{2} S_{\text {eff }} / \delta A_{\mu} \delta A_{v}$ $\left(S_{\text {eff }}=\int L_{\text {eff }} d^{2} x\right)$ is singular. We then determine the renormalized two-point fermion Green's function for all values of $a$, and study the classical field configurations $A^{\mu}$ which saturate the corresponding functional integrals. We find that, in contradistinction with the Schwinger model, the fermions exist asymptotically.

In Sec. III we construct the fermion field operators in terms of the scalar fields which were used in Ref. 1 to express the corresponding solution for the quantum field operator $A^{\mu}$. We then construct the conserved current coupling to the field tensor $F^{\mu \nu}$. The resulting equation for $F^{\mu v}$ resembles formally that of the Proca theory but with a dynamically generated mass and a nonconserved source.

The conclusions are contained in Sec. IV.

\section{GENERATING FUNCTIONALS AND FERMION GREEN'S FUNCTIONS}

We consider the cases $a>1$ and $a=1$ separately.

A. The case $a>1$

Starting from the Hamiltonian formulation developed in our earlier paper ${ }^{2}$ for $a>1$, we are led to consider the following phase-space path-integral representation of the generating functional for the Green's functions of the fields appearing in the original Lagrangian (1.1): 


$$
\begin{aligned}
Z_{a}[J, \eta, \bar{\eta}]=\mathscr{N} \int\left[\mathscr{D} A^{\mu}\right]\left[\mathscr{D} \pi_{\mu}\right][\mathscr{D} \phi]\left[\mathscr{D} \pi_{\phi}\right] \\
\times \delta\left[\Omega_{1}\right] \delta\left[\Omega_{2}\right] \prod_{x^{0}}\left[\operatorname{det}\left\{\Omega_{i}\left(x^{0}, x^{1}\right), \Omega_{j}\left(x^{0}, y^{1}\right)\right\}\right]^{1 / 2} \\
\quad \times \exp \left(i \int d^{2} x\left[\pi_{\mu} \partial_{0} A^{\mu}+\pi_{\phi} \partial_{0} \phi-\mathscr{H}_{a}\left(A^{\mu}, \pi_{\mu}, \phi, \pi_{\phi}\right)+J_{\mu} A^{\mu}\right]\right) \\
\times \exp \left(i \int d^{2} x d^{2} y \bar{\eta}(x) G(x, y ; A) \eta(y)\right),
\end{aligned}
$$

where the second-class constraints $\Omega_{1}$ and $\Omega_{2}$ are given, respectively, by

$$
\begin{aligned}
& \Omega_{1}=\pi_{0}, \\
& \Omega_{2}=\partial_{1} \pi_{1}-e^{2}(a-1) A^{0}+e^{2} A^{1}-e \pi_{\phi}-e \partial_{1} \phi .
\end{aligned}
$$

The Hamiltonian density reads

$$
\begin{aligned}
\mathscr{H}_{a}= & \frac{1}{2} \pi_{1}^{2}+\frac{1}{2} \pi_{\phi}^{2}+\frac{1}{2}\left(\partial_{1} \phi\right)^{2}+\frac{1}{2}(a-1) e^{2}\left(A^{0}\right)^{2} \\
& +\frac{1}{2}(a+1) e^{2}\left(A^{1}\right)^{2}-e\left(\partial_{1} \phi\right) A^{1}-e \pi_{\phi} A^{1}-A^{0} \Omega_{2} .
\end{aligned}
$$

The determinant appearing in (2.1) is clearly independent of the field variables and can, therefore, be absorbed into the normalization constant $N ; J_{\mu}, \bar{\eta}$, and $\eta$ are the external sources for the fields $A^{\mu}, \psi$, and $\bar{\psi}$, respectively, while $G(x, y ; A)$ is the two-point fermion Green's function in the external field $A^{\mu}$, which is easily computed to be

$$
\begin{aligned}
& G(x, y ; A) \\
& \quad=\exp \left(-2 i \sqrt{\pi} e P+\int d^{2} z A^{\mu}(z) j_{\mu}(z ; x, y)\right) S_{F}(x-y) .
\end{aligned}
$$

Here $S_{F}$ is the free fermionic propagator and $j_{\mu}$ is the "induced external current" which is given in terms of the massless Feynman propagator $D_{F}(x-y)$ by the expression

$$
j_{\mu}(z ; x, y)=\left(\partial_{\mu}^{z}+\widetilde{\partial}_{\mu}^{z}\right)\left[D_{F}(z-x)-D_{F}(z-y)\right] .
$$

Furthermore, $P_{+}$denotes the projection operator on positive-chirality states,

$$
P_{+}=\frac{1+\gamma_{5}}{2},
$$

while $\widetilde{\partial}_{\mu}$ stands for

$$
\widetilde{\partial}_{\mu}=\epsilon_{\mu \nu} \partial^{v} .
$$

By using the Fourier representation,

$$
\delta\left[\Omega_{2}\right]=\int[\mathscr{D} \xi] \exp \left(i \int d^{2} x \xi(x) \Omega_{2}(x)\right),
$$

and performing the $\pi_{0}, \pi_{\phi}$, and $A^{0}$ integrations one finds

$$
Z_{a}[J, \eta, \bar{\eta}]=\mathscr{N} \int\left[\mathscr{D} A^{\mu}\right][\mathscr{D} \phi] \exp \left(i \int d^{2} x\left(\mathscr{L}_{\mathrm{eff}}+J_{\mu} A^{\mu}\right)+i \int d^{2} x \int d^{2} y \bar{\eta}(x) G(x, y ; A) \eta(y)\right),
$$

where we have performed the relabeling $\xi=A^{0}$ and where $\mathscr{L}_{\text {eff }}(x)$ is identical with the Lagrangian density given in (1.2). Hence, in this case we obtain for $Z_{a}[0,0,0]$ the configuration space path-integral representation one would write down for an unconstrained system; i.e., the presence of constraints in $\mathscr{L}_{\text {eff }}$ does not lead to a new effective quantum action. As we shall see, this is not the case for $a=1$. Expression (2.6) also agrees with the generating functional considered in Ref. 2 and it therefore describes the quantum theory corresponding to the classical Lagrangian (1.1).

The computation of the $A^{\mu}$ propagator from (2.6) is straightforward and yields

$$
\begin{aligned}
& \left\langle 0\left|T\left(A_{\mu}(x) A_{v}(y)\right)\right| 0\right\rangle \\
& =i\left[g_{\mu \nu}+\frac{1}{a-1} \partial_{\mu} \partial_{\nu}\left(\frac{1}{e^{2}}+\frac{2}{\square}\right]\right. \\
& \left.\quad+\frac{1}{a-1} \frac{1}{\square}\left(\partial_{\mu} \widetilde{\partial}_{\nu}+\widetilde{\partial}_{\mu} \partial_{\nu}\right)\right] \Delta_{F}\left(x-y, m^{2}\right),
\end{aligned}
$$

where $\Delta_{F}\left(x-y ; m^{2}\right)$ is the free Feynman propagator for a scalar field of mass

$$
m^{2}=\frac{a^{2} e^{2}}{a-1}
$$

One also readily computes the fermion propagator by starting from (2.6) and (2.4). Needless to say, the fermions of negative chirality propagate freely [see (1.1)] while for the fermions of positive chirality one arrives at

$$
\left\langle 0\left|T\left(\psi_{+}(x) \bar{\psi}_{+}(y)\right)\right| 0\right\rangle=Z_{\psi} G_{R+}(x-y),
$$

where $G_{R+}$ is the renormalized positive-chirality fermion two-point function given by

$$
G_{R+}(x-y)=\exp \left(-\frac{4 i \pi}{a-1} \Delta_{F}\left(x-y ; m^{2}\right)\right) S_{F+}(x-y)
$$

and $Z_{\psi}$ is an ultraviolet renormalization constant 


$$
Z_{\psi}=\exp \left[\frac{4 i \pi}{a-1} \Delta_{F}\left(0 ; m^{2}\right)\right] .
$$

$S_{F+}$ denotes the free propagator for fermions of positive chirality.

From (2.10) it follows that the theory is not asymptotically free, but scales with anomalous dimension as can be seen from the short-distance behavior of $G_{R+}$ :

$$
\begin{aligned}
G_{R+}(x-y) \underset{(x-y)^{2} \rightarrow 0}{\sim}[ & {\left[-\mu^{2}(x-y)^{2}+i 0\right]^{-1 /(a-1)} } \\
& \times S_{F+}(x-y)
\end{aligned}
$$

where

$$
\mu=\frac{m}{2} e^{\gamma}
$$

with $\gamma$ the Euler constant. On the other hand, for $(x-y)^{2} \rightarrow-\infty$ one has

$$
G_{R+}(x-y) \underset{(x-y)^{2} \rightarrow-\infty}{\sim} S_{F+}(x-y)
$$

which shows that, in contradistinction with the Schwinger model, the fermions exist asymptotically. Furthermore, from (2.13) one sees that the Green's function $G_{R}+$ clusters. This is also true for the $n$-point fermion Green's function, and, hence, there is no vacuum degeneracy. This is not in contradiction with the fact that the Euclidean functional integral corresponding to $G_{R}+$ is saturated by the field configuration $A_{\mu}^{\mathrm{cl}}(z ; x, y)$ which decomposes into two parts centered around $x$ and $y$, respectively,

$$
A_{\mu}^{\mathrm{cl}}(z ; x, y)=A_{\mu}^{[1 / a]}(z ; x)+A^{[-1 / a]}(z ; y),
$$

where each part $\left(\epsilon_{12}=1\right)$

$$
\begin{aligned}
& A_{\mu}^{[ \pm 1 / a]}(z ; \xi) \\
& = \pm \frac{1}{e}\left[-\frac{2 \sqrt{\pi}}{a}\left(\partial_{\mu}+\widetilde{\partial}_{\mu}\right)\left[\Delta\left(z-\xi ; m^{2}\right)-D(z-\xi)\right]\right. \\
& \left.\quad+\frac{2 \sqrt{\pi}}{a-1} \partial_{\mu} \Delta\left(z-\xi ; m^{2}\right)\right]
\end{aligned}
$$

carries a nontrivial "winding number"

$$
e \sqrt{\pi} \int \frac{d^{2} x}{4 \pi} \epsilon_{\mu \nu} F_{\mu v}^{[ \pm 1 / a]}= \pm \frac{1}{a} .
$$

Indeed, although $A_{\mu}^{[ \pm 1 / a]}$ tends to "pure gauge" at infinity,

$$
\begin{aligned}
& A_{\mu}^{[ \pm 1 / a]}(z ; \xi) \underset{z \rightarrow \infty}{\rightarrow} \pm \frac{1}{e \sqrt{\pi}} \partial_{\mu} \Theta \\
& \Theta=\frac{1}{2 \sqrt{\pi} a} \ln \left(\mu^{2} z^{2}\right)+\frac{1}{a} \arctan \frac{z_{2}}{z_{1}},
\end{aligned}
$$

one must keep in mind that one is no longer dealing with a gauge theory; that is, pure gauge does not correspond here to a vacuum configuration and, therefore, the existence of a nontrivial winding number has no implications on the vacuum structure. ${ }^{6}$

\section{B. The case $a=1$}

For $a=1$, the generating functional (2.6) does not define a bona fide quantum theory, since the operator $\Omega_{\mu v}$ defined through

$$
\begin{array}{r}
\int[\mathscr{D} \phi]\left[\mathscr{D} A^{\mu}\right] \exp \left(i \int d^{2} x \mathscr{L}_{\mathrm{eff}}\right) \\
=\int\left[\mathscr{D} A^{\mu}\right] \exp \left(\frac{i}{2} \int d^{2} x d^{2} y A^{\mu}(x)\right. \\
\left.\times \Omega_{\mu \nu}(x, y) A^{v}(y)\right)
\end{array}
$$

is no longer an invertible operator. Nevertheless, we can still obtain a configuration space path-integral representation for $Z_{a=1}$ by starting again from the corresponding phase-space path integral associated with the constrained Hamiltonian dynamics for $a=1$, which was presented in Ref. 1. One has

$$
\begin{aligned}
Z_{a=1}[J, \eta, \bar{\eta}]=\mathscr{N} \int\left[\mathscr{D} A^{\mu}\right]\left[\mathscr{D} \pi_{\mu}\right][\mathscr{D} \phi]\left[\mathscr{D} \pi_{\phi}\right] \\
\times \prod_{i=1}^{4} \delta\left[\Omega_{i}\right] \exp \int i \int d^{2} x\left[\pi_{\mu} \partial_{0} A^{\mu}+\pi_{\phi} \partial_{0} \phi-\mathscr{H}_{a=1}\left(A^{\mu}, \pi_{\mu}, \phi, \pi_{\phi}\right)+J_{\mu} A^{\mu}\right) \\
\quad \times \exp \left(i \int d^{2} x d^{2} y \bar{\eta}(x) G(x, y ; A) \eta(y)\right),
\end{aligned}
$$

where the constraints now are

$$
\begin{aligned}
& \Omega_{1}=\pi_{0}, \\
& \Omega_{2}=\partial^{1} \pi_{1}-e\left(e A^{1}-\pi_{\phi}+\partial^{1} \phi\right), \\
& \Omega_{3}=\pi_{1}, \\
& \Omega_{4}=e\left(A^{0}+A^{1}\right)+\left(e A^{1}-\pi_{\phi}+\partial^{1} \phi\right) .
\end{aligned}
$$

From (2.18) it follows that the Faddeev-Popov determinant is again field independent and hence can be absorbed into the normalization constant $N$. Notice the drastic change in the constraint structure that has occurred when going from the case $a>1$ to $a=1$. By carrying out all functional integrations in (2.17), one arrives at

$$
\begin{array}{r}
Z_{a}[J, \eta, \bar{\eta}]=\exp \left[i \int d^{2} x d^{2} y \bar{\eta}(x)\right. \\
\left.\times G\left[x, y ; \frac{1}{i} \frac{\delta}{\delta J_{\mu}}\right) \eta(y)\right] Z[J],
\end{array}
$$


where $\left[\partial_{+}=\frac{1}{2}\left(\partial_{0}+\partial_{1}\right)\right]$,

$$
\begin{aligned}
Z[J]=\exp \left[\frac{i}{2} \int\right. & d^{2} x d^{2} y\left(J_{0}-J_{1}\right)(x) \frac{1}{e^{2}}\left(\square-4 \partial_{+} \partial_{+}\right) \\
& \left.\times D_{F}(x-y)\left(J_{0}-J_{1}\right)(y)\right)
\end{aligned}
$$

From here we deduce the $A^{\mu}$ propagator:

$$
\begin{aligned}
\left\langle 0\left|T\left(A^{\mu}(x) A^{v}(y)\right)\right| 0\right\rangle & \\
= & \frac{i}{e^{2}}\left(\partial^{\mu}+\widetilde{\partial}^{\mu}\right)\left(\partial^{v}+\widetilde{\partial}^{v}\right) D_{F}(x-y) \\
& -\frac{i}{e^{2}}\left(g^{\mu 0}+e^{\mu 0}\right)\left(g^{\nu 0}+\epsilon^{\nu 0}\right) \delta^{(2)}(x-y) .
\end{aligned}
$$

It is obvious from (2.20) that the $A^{\mu}$ propagator respects the constraint $A^{0}=-A^{1}$ following from (2.18). Furthermore, one easily verifies that (2.20) agrees with the result obtained from the operator solution and the commutation rules presented in Ref. 1. One can also check that (2.20) respects the constraint $\pi_{1}=F^{01}=0$.

It is remarkable that the above propagator $(2.20)$ does not possess an inverse, not even after deleting the noncovariant contact terms, and therefore cannot be obtained from any effective Lagrangian. Following common practice we define the $A^{\mu}$ propagator in terms of the $T^{*}$ product obtained by dropping the noncovariant contact terms in (2.20). Then

$$
\begin{aligned}
& \left\langle 0\left|T^{*}\left(A^{\mu}(x) A^{v}(y)\right)\right| 0\right\rangle \\
& =\frac{i}{e^{2}}\left(\partial^{\mu}+\widetilde{\partial}^{\mu}\right)\left(\partial^{v}+\widetilde{\partial}^{v}\right) D_{F}(x-y) .
\end{aligned}
$$

This expression still respect the constraints $A^{0}=-A^{1}$ for all $x$ and $y$, as well as $\pi_{1}=F^{01}=0$ for $x^{0} \neq y^{0}$.

The Fermion propagator can now be readily computed from (2.19), the result being

$$
\langle 0|T(\psi(x) \bar{\psi}(y))| 0\rangle=i S_{F}(x-y) .
$$

Hence, for $a=1$, fermions of both chiralities propagate freely not being affected at all by the interaction. This is a surprising result which will be elucidated in the next section.

\section{BOSONIZATION OF THE FERMION FIELD}

In Ref. 1 we found the operator solution for $A^{\mu}$ in terms of scalar fields for the cases $a>1$ and $a=1$. In this section we shall complete this work by providing the corresponding operator realization for the fermion field $\psi$. Again we treat the cases $a>1$ and $a=1$ separately.

\section{A. The case $a>1$}

In Refs. 2 and 1 it was shown that

$A^{\mu}(x)=-\frac{1}{a e}\left[\left(\partial^{\mu}+\widetilde{\partial}^{\mu}\right) \sigma-a \widetilde{\partial}^{\mu} \sigma-\left(\partial^{\mu}+\widetilde{\partial}^{\mu}\right) h\right]$,

where $\sigma$ is a free scalar field of mass $m$ satisfying the equal-time commutation relation

$$
\left[\sigma(x), \partial_{0} \sigma(y)\right]_{x^{0}=y^{0}}=\frac{i}{a-1} \delta\left(x^{1}-y^{1}\right)
$$

while $h$ is a massless canonical scalar field.

The operator $\psi(x)$ is obtained by solving the Dirac equation

$$
\begin{aligned}
i \gamma^{\mu} \partial_{\mu} \psi(x)+e \sqrt{\pi} \gamma^{\mu} P+\lim _{\substack{\epsilon \rightarrow 0 \\
\epsilon^{2}<0}}[ & A_{\mu}(x+\epsilon) \psi(x) \\
& \left.\quad+\psi(x) A_{\mu}(x-\epsilon)\right]=0,
\end{aligned}
$$

where $A_{\mu}$ is given by (3.1). For the positive- and negative-chirality components $\psi_{ \pm}$Eq. (3.3) reduces to

$$
\begin{aligned}
& i \gamma^{\mu} \partial_{\mu} \psi_{+}-\sqrt{\pi} \gamma^{\mu} \lim _{\substack{\epsilon \rightarrow 0 \\
\epsilon^{2}<0}}\left[\partial_{\mu} \sigma(x+\epsilon) \psi_{+}(x)\right. \\
&\left.+\psi_{+}(x) \partial_{\mu} \sigma(x-\epsilon)\right]=0,
\end{aligned}
$$

$i \gamma^{\mu} \partial_{\mu} \psi_{-}=0$

The renormalized solutions read

$$
\begin{aligned}
& \psi_{+}(x)=: e^{-2 i \sqrt{\pi} \sigma(x)}: \psi_{+}(x)_{f}, \\
& \psi_{-}(x)=\psi_{-}(x)_{f} .
\end{aligned}
$$

Notice that, since the field $h$ no longer appears in the equations of motion (3.4), there exists no a priori relation between the free fermion field $\psi_{f}$ and $h$. Nevertheless, the consistency of the theory demands, as we shall see, that $\psi(x)_{f}$ be given by the Mandelstam bosonization formula $(\tilde{\mu}$ is an infrared regulator):

$$
\psi_{\alpha}(x)_{f}=\left[\frac{\tilde{\mu}}{2 \pi}\right]^{1 / 2} \exp \left[-\frac{i \pi}{4}\left(\gamma_{5}\right)_{\alpha \alpha}\right]: \exp \left(i \sqrt{\pi}\left(\gamma_{5}\right)_{\alpha \alpha} h(x)+i \sqrt{\pi} \int_{x^{1}}^{\infty} d y^{1} \partial_{0} h\left(x^{0}, y^{1}\right)\right]: \text {. }
$$

With the aid of the commutation relations (3.2) one easily verifies that the solution (3.5) leads to the renormalized fermion two-point function (2.10).

We next address ourselves to the problem of constructing a conserved current acting as the source of $F^{\mu \nu}$. The most obvious candidate would be

$$
j_{+}^{\mu}(x)=-2 e \sqrt{\pi} \lim _{\substack{\epsilon \rightarrow 0 \\ \epsilon^{2}<0}} Z^{-1}(\epsilon)\left[\psi_{+}^{\dagger}(x+\epsilon) \gamma^{0} \gamma^{\mu} \exp \left[i e \sqrt{\pi} \int_{x}^{x+\epsilon} d z^{\mu} B_{\mu}(z)\right) \psi_{+}(x)\right]-\mathrm{VEV},
$$

where VEV stands for "vacuum expectation value," $Z(\epsilon)$ is a renormalization constant for the composite operator to be 
determined at a later stage, and $\left(\widetilde{A}^{\mu}=\epsilon^{\mu v} A_{v}\right)$

$$
B^{\mu}(z)=\kappa_{1} A^{\mu}(z)+\kappa_{2} \widetilde{A}^{\mu}(z) .
$$

For the sake of generality we have introduced two undetermined constants, $\kappa_{1}$ and $\kappa_{2}$, in the line integral; the choice $\kappa_{1}=-\kappa_{2}=1$ formally corresponds to a "gauge-invariant" regularization of the operator product in (3.7). Using (3.5a), one finds

$j_{+}^{\mu}(x)=-2 e \sqrt{\pi}\left\{: \bar{\psi}_{+}(x)_{f} \gamma^{\mu} \psi_{+}(x)_{f}:+\frac{\left(\kappa_{1}+\kappa_{2}\right)}{2 \sqrt{\pi} a}\left(\partial^{\mu}+\widetilde{\partial}^{\mu}\right) h(x)+\frac{1}{2 \sqrt{\pi}}\left[1-\left[\frac{2-a}{2 a}\right]\left(\kappa_{1}+\kappa_{2}\right)\right]\left(\partial^{\mu}+\widetilde{\partial}^{\mu}\right) \sigma(x)\right\}$,

For arriving at (3.9) we have chosen $Z(\epsilon)$ such as to yield the standard form for the free part of the chiral current, that is

$$
Z(\epsilon)=\left(\frac{1}{\left(-m^{2} \epsilon^{2}\right)}\right)^{1 /(a-1)}
$$

Since $\sigma(x)$ is a massive free field, it is evident that $j_{+}^{\mu}$ is not conserved for the formally gauge-invariant regularization prescription $\kappa_{1}=-\kappa_{2}=1$. In fact, conservation would be achieved for the choice

$$
\kappa_{1}+\kappa_{2}=\frac{2 a}{2-a} \text {. }
$$

However, for this choice the current will be singular for $a=2$ and hence cannot be the source of $F^{\mu \nu}$. Indeed, from (3.1) it follows that the conserved chiral current coupling to $F^{\mu v}$ defined by

$$
J_{+}^{v} \equiv \partial_{\mu} F^{\mu v}
$$

is given by

$$
J_{+}^{v}=-e a \widetilde{\partial}^{v} \sigma .
$$

We now show how this current can be constructed in terms of the fundamental fields $\psi$ and $A^{\mu}$. To this end we consider

$$
\begin{aligned}
j_{+}^{\mu}(x)-e^{2} a b A^{\mu}(x)=-2 e \sqrt{\pi}[ & : \bar{\psi}_{+}(x)_{f} \gamma^{\mu} \psi_{+}(x)_{f}:+\frac{1}{2 \sqrt{\pi}}\left(1-b-\frac{2-a}{2 a}\left(\kappa_{1}+\kappa_{2}\right)\right]\left(\partial^{\mu}+\widetilde{\partial}^{\mu}\right) \sigma(x) \\
& \left.+\frac{1}{2 \sqrt{\pi}}\left[b+\frac{1}{a}\left(\kappa_{1}+\kappa_{2}\right)\right]\left(\partial^{\mu}+\widetilde{\partial}^{\mu}\right) h(x)+\frac{a b}{2 \sqrt{\pi}} \widetilde{\partial}^{\mu} \sigma(x)\right]
\end{aligned}
$$

which follows from (3.9) and (3.1). Here $b$ is a so-far undetermined constant. In order for (3.14) to reduce to (3.13), we must choose $b=1, \kappa_{1}=-\kappa_{2}$, and $\psi_{f}$ to be given in terms of the harmonic field $h$ by the Mandelstam representation (3.6). We then identify

$$
J_{+}^{\mu}(x)=j_{+}^{\mu}(x)-e^{2} a A^{\mu}(x)
$$

with the conserved current coupling to $F^{\mu \nu}$. Thus, the classical equation of motion $\partial_{\mu} F^{\mu v}=-2 e \sqrt{\pi} \bar{\psi}_{+} \gamma^{v} \psi_{+}$, following from (1.2) is replaced at quantum level by

$$
\partial_{\mu} F^{\mu v}(x)+e^{2} a A^{v}(x)=j_{+}^{v}(x),
$$

which explicitly displays the dynamical breakdown of gauge invariance. We emphasize again that $j_{+}^{v}$ in (3.16) is the nonconserved current (3.9) evaluated for $\kappa_{1}=-\kappa_{2}$. Although the local chiral symmetry is broken, the current $J_{+}^{\mu}$, coupling to $F^{\mu \nu}$, is conserved which suggests that the global chiral symmetry remains unbroken. However, the chiral-charge operator $Q_{+}$constructed from $J_{+}^{0}$ commutes with $\psi_{+}$, as seen from (3.5a) and (3.2). Hence $\psi_{+}$is chirally neutral, much as in the Schwinger model ${ }^{7}$ where the (gauge-invariant) fermion operator is electrically neutral. ${ }^{8,9}$ Nevertheless, the operator $\psi_{+}$carries a chiral quantum number associated with the conserved free-chiral charge $q_{+}$,

$$
q_{+}=-2 e \sqrt{\pi} \int d x^{1}: \bar{\psi}_{+}(x)_{f} \gamma^{0} \psi_{+}(x)_{f}: .
$$

Indeed,

$$
\left[q_{+}, \psi_{+}(x)\right]=2 e \sqrt{\pi} \psi_{+}(x) .
$$

In other words, $\psi_{+}$remembers the chirality of the noninteracting field. Since $Q_{+}$and $q_{+}$commute with the Hamiltonian and annihilate the vacuum, there is no spontaneous breakdown of the global chiral symmetry and the operator $q_{+}$provides the usual chiral selection rules for the fermionic Green's functions.

\section{B. The case $a=1$}

In Ref. 1 it was shown that for $a=1$

$$
A^{\mu}(x)=-\frac{1}{e}\left(\partial^{\mu}+\widetilde{\partial}^{\mu}\right) \phi(x),
$$

where $\phi(x)$ is a free canonical massless scalar field. Substituting (3.19) into (3.3), one finds

$$
\psi=\psi_{f}
$$

which confirms the result (2.22) obtained within the func- 
tional approach. However, since (3.19) implies $F^{\mu v}=0$, the free fermionic current cannot be the source of $F^{\mu v}$. Interestingly enough, the combination

$$
J_{+}^{\mu}(x)=j_{+}^{\mu}(x)_{f}-e^{2} A^{\mu}(x),
$$

suggested by (3.15), vanishes identically if the identification $h=\phi$ is made in the Mandelstam representation (3.6). This leads us to conjecture that for $a>1$ and $a=1$ the underlying quantum equation of motion for $F^{\mu \nu}$ is of the form

$$
\partial_{\mu} F^{\mu v}(x)+e^{2} a A^{v}(x)=N\left[\bar{\psi}_{+}(x) \gamma^{v} \psi_{+}(x)\right],
$$

where the right-hand side is generally defined by the limiting procedure (3.7) with $\kappa_{1}=-\kappa_{2}$. The nonsmooth character of the limit $a \rightarrow 1$, emphasized in Ref. 1, now manifests itself in the singular transition $j_{+}^{\mu}(x) \rightarrow j_{+}^{\mu}(x)_{f}$, or, equivalently, in the singular behavior of $\psi$ for $a \rightarrow 1$ [see (3.5a) and (2.10)].

\section{CONCLUSIONS}

In this paper we have used the effective Lagrangian (1.2) in order to study the quantum dynamics of the chiral fermion fields defined by (1.1). This was done by using functional as well as operator methods.

For $a>1$ the two-point fermion Green's functions were found to scale with anomalous dimension and shown to cluster for large spacelike separations. There is no vacuum degeneracy and no spontaneous breakdown of the global chiral symmetry. In terms of $\psi$ and $A^{\mu}$ the quantum theory is described by the covariant Dirac equation and a Proca-type equation for $F^{\mu v}$ with a dynamically generated mass and a nonconserved source.

For $a=1$, the fermions of both chiralities propagate freely. An interesting feature of this case is that the configuration space representation of the generating functional $Z_{a=1}$ cannot be obtained as the limit $a \rightarrow 1$ of $Z_{a}$, and in fact cannot be written in terms of an effective Lorentzinvariant Lagrangian. This reflects the sudden change in the constraint structure of the theory as $a \rightarrow 1$.

\section{ACKNOWLEDGMENT}

One of the authors (H.O.G.) was supported in part by Conselho Nacional de Desenvolvimento Cientifico e Tecnologico (CNPq), Brazil.
*On leave of absence from Instituto de Fisica, Universidade Federal do Rio Grande do Sul, 90000 Porto Alegre, RS, Brazil.

${ }^{1}$ H. O. Girotti, H. J. Rothe, and K. D. Rothe, Phys. Rev. D 33, 514 (1986); also see R. Rajaraman, Phys. Lett. 154B, 305 (1985); CERN Report No. CERN-TH 4268, 1985 (unpublished).

${ }^{2}$ R. Jackiw and R. Rajaraman, Phys. Rev. Lett. 54, 1219 (1985).

${ }^{3}$ Our conventions are $g^{00}=-g^{11}=1, \epsilon^{01}=1, \gamma_{\mu} \gamma^{5}=\epsilon_{\mu \nu} \gamma^{\nu}$.

${ }^{4}$ E. S. Fradkin and G. A. Vilkovisky, CERN Report No. CERN-TH 2332, 1977 (unpublished).
${ }^{5}$ P. Senjanovic, Ann. Phys. (N.Y.) 100, 227 (1976).

${ }^{6}$ The connection between "induced instantons" and the vacuum structure in $\mathrm{QED}_{2}$ was studied by N. K. Nielsen and B. Schroer, Nucl. Phys. B120, 62 (1979); K. D. Rothe and J. A. Swieca, Ann. Phys. (N.Y.) 117, 382 (1979).

7J. Schwinger, Phys. Rev. 128, 2425 (1962).

8J. H. Lowenstein and J. A. Swieca, Ann. Phys. (N.Y.) 68, 172 (1971).

${ }^{9}$ L. V. Belvedere, J. A. Swieca, K. D. Rothe, and B. Schroer, Nucl. Phys. B153, 112 (1979). 